OPEN ACCESS

Edited by:

Pedro Gamito,

Universidade Lusófona, Portugal

Reviewed by:

Per Møller,

University of Copenhagen, Denmark

Mickael L. D. Deroche,

Concordia University, Canada

Akitoshi Ogawa,

Juntendo University, Japan

*Correspondence:

Arash Yazdanbakhsh

yazdan@bu.edu

Specialty section:

This article was submitted to

Cognitive Science,

a section of the journal

Frontiers in Psychology

Received: 17 July 2019

Accepted: 18 December 2019

Published: 22 January 2020

Citation:

Ogmen $H$, Shibata $K$ and Yazdanbakhsh A (2020) Perception,

Cognition, and Action

in Hyperspaces: Implications on Brain

Plasticity, Learning, and Cognition.

Front. Psychol. 10:3000

doi: 10.3389/fpsyg.2019.03000

\section{Perception, Cognition, and Action in Hyperspaces: Implications on Brain Plasticity, Learning, and Cognition}

\author{
Haluk Ogmen ${ }^{1}$ Kazuhisa Shibata ${ }^{2}$ and Arash Yazdanbakhsh ${ }^{3 *}$ \\ ${ }^{1}$ Laboratory of Perceptual and Cognitive Dynamics, Electrical \& Computer Engineering, Ritchie School of Engineering \& \\ Computer Science, University of Denver, Denver, CO, United States, ${ }^{2}$ Laboratory for Human Cognition and Learning, RIKEN \\ Center for Brain Science, Wako, Japan, ${ }^{3}$ Department of Psychological and Brain Sciences, Computational Neuroscience \\ and Vision Lab, Center for Systems Neuroscience, Boston University, Boston, MA, United States
}

We live in a three-dimensional (3D) spatial world; however, our retinas receive a pair of 2D projections of the 3D environment. By using multiple cues, such as disparity, motion parallax, perspective, our brains can construct 3D representations of the world from the 2D projections on our retinas. These 3D representations underlie our 3D perceptions of the world and are mapped into our motor systems to generate accurate sensorimotor behaviors. Three-dimensional perceptual and sensorimotor capabilities emerge during development: the physiology of the growing baby changes hence necessitating an ongoing re-adaptation of the mapping between 3D sensory representations and the motor coordinates. This adaptation continues in adulthood and is quite general to successfully deal with joint-space changes (longer arms due to growth), skull and eye size changes (and still being able of accurate eye movements), etc. A fundamental question is whether our brains are inherently limited to $3 \mathrm{D}$ representations of the environment because we are living in a 3D world, or alternatively, our brains may have the inherent capability and plasticity of representing arbitrary dimensions; however, 3D representations emerge from the fact that our development and learning take place in a 3D world. Here, we review research related to inherent capabilities and limitations of brain plasticity in terms of its spatial representations and discuss whether with appropriate training, humans can build perceptual and sensorimotor representations of spatial 4D environments, and how the presence or lack of ability of a solid and direct $4 \mathrm{D}$ representation can reveal underlying neural representations of space.

Keywords: hypercube, brain plasticity, sensorimotor, four-dimensional, neural representations, learning, brain development

\section{INTRODUCTION}

Species operate in space and their behavioral success depends on how well they process, represent, store, and recall spatial information. In addition to being a fundamental aspect of sensorimotor behavior, the concept of space plays an important role in our understanding of higher cognitive functions. Indeed, the concept of space has been central to the thinking of many philosophers such as Plato, AlHazen, Descartes, Hume, and Kant to name a few (Huggett, 1999). Questions such as whether space is a priori or learned, whether it is objective or subjective have been debated. The concepts of space and time and their relationship shape theories in modern physics. Given this background, it goes without saying that the concept of space also plays a major role in psychology and neuroscience. For example, based on children's spatial reorientation and 
navigation behavior, Spelke et al. (2011) concluded that two systems of human core knowledge (i.e., ancient, innate, and universal systems) reflect the primary properties of Euclidian plane geometry. She suggested that these systems lack the power of abstract generalization; however, language and symbolic representations allow children to combine productively their core knowledge to attain abstract thoughts that form the foundations of abstract mathematical geometry (Spelke et al., 2011). According to the Piagetian theory, higher cognitive functions are built upon sensorimotor schema, which represent the operation of sensory and motor systems in space and time (Piaget, 1936, 1950). The progression from sensorimotor stage to formal operational stage takes place as ego-centric "subjective" sensorimotor representations, i.e., representations whose reference frames are based on the subject, such as head-centered representations, become coordinated across initially independent sensory spaces (e.g., ego-centric visual space, ego-centric tactile space) to give rise to exo-centric (i.e., reference-frames) based outside of the subject "objective" representations (e.g., object-centered representations) (Piaget, 1936, 1950). Neurophysiological studies revealed multiple representations of space in various brain areas, including egocentric (Gross and Graziano, 1995; Grieves and Jeffery, 2017) and exo-centric representations (Olson, 2003). Notwithstanding specific theoretical stances and underlying neurophysiological correlates, these approaches focus on three-dimensional (3D) or two-dimensional (2D) representations simply because our environment is $3 \mathrm{D}$ and its projections are $2 \mathrm{D}$. Our visual system receives on our retinas a pair of $2 \mathrm{D}$ projections of a 3D environment. By using multiple cues, such as disparity, motion parallax, perspective, and shading, our brains can construct $3 \mathrm{D}$ representations of the world from its $2 \mathrm{D}$ projections on our retinas.

A natural question that arises is, if our brains can construct $3 \mathrm{D}$ representations from the $2 \mathrm{D}$ projections (Figure 1A), can it also construct $4 \mathrm{D}$ spatial representations from $2 \mathrm{D}$ projections that represent a spatially 4D world (Figure 1B)? This question touches a fundamental theme regarding the limits of plasticity in the brain: Are our brains hard-wired to be limited to
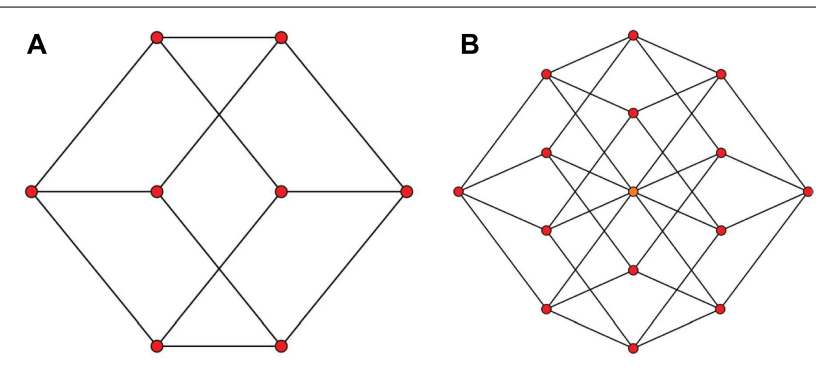

FIGURE 1 | Two-dimensional projections of a 3D and a 4D object. It is easy and accessible for us to have a solid 3D impression of (A) as a cube, however, looking at (B) doesn't pop a solid 4D impression of a hypercube. Panels (A,B) are from: http://en.m.wikipedia.org/wiki/File:3-cube column graph.svg and http://commonswikimedia.org/wiki/File:4-cubecolumngraph.svg, respectively, and have been released into the public domain by their author Geoff Richards (Qef).
$3 \mathrm{D}$ representations because it evolved and developed in $3 \mathrm{D}$ worlds, or do they have inherent plasticity to represent arbitrary dimensions? The neurophysiology and neuroanatomy of the brain indicate high-dimensional internal representations: For example, the representation for an object not only includes three-dimensional space but also other feature dimensions like brightness, hue, texture, curvature, etc. However, whether these internal high-dimensional representations can be translated to higher dimensions for the external space remains unanswered. Support for brain plasticity in spatial representations comes from inherent plasticity that allows to cope for natural changes that occur, in particular, during the developmental period where three-dimensional perceptual and sensorimotor capabilities emerge. A fundamental aspect of development is that the physiology of the growing baby changes hence necessitating an ongoing re-adaptation of the $3 \mathrm{D}$ representations and the mapping between $3 \mathrm{D}$ sensory representations and the motor coordinates. This adaptation continues in adulthood. But is this adaptation general to deal with drastic changes such as going from $3 \mathrm{D}$ to $4 \mathrm{D}$ ? Hence, a fundamental question is whether our brains are inherently limited to $3 \mathrm{D}$ representations of the environment because we are living in a 3D world. Alternatively, our brains may have the inherent capability of representing arbitrary dimensions; however, $3 \mathrm{D}$ representations emerge from the fact that our development and learning take place in a $3 \mathrm{D}$ world.

\section{OPERATIONALIZATION OF THE CONCEPT}

Studies exploring 4D environments focused on different aspects of the underlying concept. For example, the titles of these studies refer to the phenomenon under study as " $4 D$ spatial reasoning," " $4 D$ spatial intuition," " $4 D$ spatial judgments." " $4 D$ spatial representations." They also use different variables to operationalize the concept. Such a diversity is expected because our spatial competencies are multi-faceted and have different neural correlates. We can perceive three-dimensional objects, we can reason about three-dimensional space and objects in abstract ways, and we can navigate in the $3 \mathrm{D}$ space. Although these abilities do have some communalities, their underlying computations and neural correlates are not identical. For example, when we state that we perceive a $3 \mathrm{D}$ object, we may be referring to the perceptions that arise from a $2 \mathrm{D}$ drawing with perspective cues versus those that arise in a virtual reality headset. Although in both cases we "perceive a 3D structure," the phenomenal experience from a 2D drawing vs. from virtual reality are extremely different suggesting differences in their neural representations.

\section{SUMMARY OF FINDINGS}

Aflalo and Graziano (2008) studied subjects' path-integration ability in 4D environments to assess their "four-dimensional spatial reasoning." Subjects (five adults) were asked to navigate 
in a $4 \mathrm{D}$ maze and, upon reaching the end of the maze, to point to the location of the starting point of the maze. The angular difference between subjects' response and the true direction quantified the error in path integration. The 4D maze was constructed by mapping the $4 \mathrm{D}$ stimulus on a $2 \mathrm{D}$ display through projective geometry (this is called the "projection technique" of $4 \mathrm{D}$ stimulus generation). An additional cue was added for the fourth dimension: The extremes of the fourth dimension's axis $( \pm \infty)$ were labeled "hot" and "cold" and hues between red and blue were used to signal to the subject where s/he is along the fourth dimension's axis. Subjects were allowed to practice on their own computer at home and spent $15 \mathrm{~min}$ to $1 \mathrm{~h}$ per day for several weeks. They were given feedback after each trial. During the initial trials in laboratory testing for the $4 \mathrm{D}$ maze, subjects' performance improved rapidly (ca. 20 trials; average trial duration ranged from $45 \mathrm{~s}$ to $2.7 \mathrm{~min}$ ) and remained thereafter at a plateau level. A second improvement in performance was observed after the plateau phase (ca. 160 trials). Aflalo and Graziano (2008) compared their subjects' performance to those of two theoretical (simulated) models that were designed to solve the puzzle by using only 3D spatial reasoning. With subjects performing better than these models, Aflalo and Graziano (2008) concluded that subjects were capable of $4 \mathrm{D}$ spatial reasoning, as assessed by the path-integration task.

Ambinder et al. (2009) used 4D tetrahedrons and presented subjects successive slices of the stimuli as it would happen if the subject were to walk through the fourth dimension's axis (this is called the "slicing technique" of $4 \mathrm{D}$ stimulus generation). Observers (four adults) judged the distance between two vertices or the orientation of a surface plane. No feedback was given. They found a significant positive correlation between reported distances/orientations and the true 4D distances/orientations (among conditions and subjects, $R^{2}$ values ranged from 0.29 to 0.81 ). They concluded that humans can represent and make geometrical judgments (distance and orientation) about $4 \mathrm{D}$ objects.

In a follow-up study, Wang (2014a) noted that the pathintegration task used by Aflalo and Graziano (2008) can be solved by using exclusively $3 \mathrm{D}$ information. There is also evidence that navigation tasks may be based on graphs rather than geometrical maps (Chrastil and Warren, 2014). Wang (2014a) also pointed out that length and orientation judgments used by Ambinder et al. (2009) are 1D and 2D geometric properties, respectively. To eliminate these lower dimensional solutions, she selected a $4 \mathrm{D}$ property, the hyper-volume of $4 \mathrm{D}$ objects, as the dependent variable. To present the $4 \mathrm{D}$ stimuli, she used a "rotation technique," where the $4 \mathrm{D}$ object is rotated around a selected plane. No feedback was given to the subjects (three adults). Results showed significant correlations between reported and actual hypervolumes ( $\beta$ values ranged from 0.44 to 0.73 ) and Wang concluded that humans can have $4 \mathrm{D}$ representations that provide estimates of inherently 4D properties, such as hyper-volume.

Miwa et al. (2018) designed an interactive environment wherein a $4 \mathrm{D}$ hypercube was projected to a $3 \mathrm{D}$ space by using perspective projection. A hypercube was centered at the origin of the $4 \mathrm{D}$ space and each of the eight sides of the $4 \mathrm{D}$ cube was displayed with a different color. Perspective projection makes parallel lines converge at infinity ${ }^{1}$ and this point of convergence is called the "vanishing point." When the parallel lines are also parallel to one of the axes of the coordinate system, these points are called "principal vanishing points." These principal vanishing points were displayed along with the stimulus. The observer could change her point of view of the stimulus by manipulating principal vanishing points. This allowed the observer to actively visualize and explore the stimulus. Before the start of the main experiment, observers were given up to $3 \mathrm{~h}$ to actively visualize and explore the stimulus. They were then tested on their ability to navigate along checkpoints in the $4 \mathrm{D}$ space. These checkpoints were chosen randomly with the constraint that three or four sides of the hypercube are simultaneously visible from the checkpoint. Of their 12 subjects (mean age: 20.5 years), one dropped out, three had poor performance (measured by the proportion of correctly visited checkpoints) and the remaining eight had successful performance $(\geq 70 \%)$. In a second experiment, they used the so-called "color cube test," where an $N$-dimensional cube is constructed with each side having a different color. This cube is then projected to a $N-1$ dimensional space and observers are shown $N$ different views of the cube in the $N-1$ dimensional space. The task of the observer was to answer questions like "what color is the side of the cube which is positioned opposite to the green side?" To accomplish this task in 3D, observers typically mentally rotate a $3 \mathrm{D}$ mental representation of the cube to understand the relationships between different sides/colors. Of the twelve subjects, two dropped out, three had poor performance whereas the remaining seven had good performance ( $\geq 70 \%)$. Based on these results, the authors concluded that "humans are capable of learning 4D representations."

While higher order visual areas are involved in $3 \mathrm{D}$ vision (Tsao et al., 2003), neural responses in early visual areas are modulated by stimulus 3D configuration (Dobbins et al., 1998). Qian and Yazdanbakhsh (2015), suggested a neural model for interactions between higher and lower visual areas encoding distance and size that can be one of bases of size constancy - perceiving an object size irrespective of its distance, as one of the lower order spatial recognition processes. As such, if human direct 4D percept materializes, we may reach to broader forms of visual object constancy that signify higher order scene groupings to be reflected by modern artists and virtual reality developers.

\section{OUTSTANDING PROBLEMS: OPERATIONALIZATION OF THE CONCEPT}

As mentioned previously, human perception, cognition, and action are multi-faceted. When we study human perception, cognition, and action in $3 \mathrm{D}$, we introduce numerous experimental paradigms and variables intended to capture different aspects of brain processes and behaviors. Similarly, studies of $4 \mathrm{D}$ used different approaches such as navigation

\footnotetext{
${ }^{1}$ With the exception of those lines that are parallel to the projection plane; these
} lines remain parallel under perspective projection. 
performance and a variety of geometric judgments. Adaptation experiments with inverting glasses (Stratton, 1897; Kohler, 1962) provide a good illustration why one needs to examine different variables: In these experiments, subjects were fitted with inverting glasses making the stimuli appear "upside down." First, subjects had difficulty in operating with these glasses but with practice they became quite adept in complex behaviors, such as riding a bicycle in city streets. However, in general, subjects did not experience the stimulus flipping to its normal, instead of upside-down appearance. Hence, at the perceptual level, the system did not compensate for and correct the effect of inverting glasses but it did so at the sensorimotor level (Harris, 1965; Linden et al., 1999). Hence, careful definitions of dependent and independent variables to reflect the multi-faceted aspects of our brain processes and behaviors are important to explore $4 \mathrm{D}$ performance. Random dot stereograms have been used to generate $3 \mathrm{D}$ phenomenal experience from $2 \mathrm{D}$ images without any information other than disparity. An adaptation of this approach for $4 \mathrm{D}$ percepts may help generate phenomenological $4 \mathrm{D}$ experience. How this $4 \mathrm{D}$ perception would be is difficult to answer; however, we can extrapolate our experiences that arise when we perceive $3 \mathrm{D}$ structures in random dot stereograms or the experience in a $3 \mathrm{D}$ movie theater with and without polarizing glasses.

\section{OUTSTANDING PROBLEMS: GENERATION OF 4D STIMULI IN A 3D WORLD}

Methods for presenting four and higher dimensional stimuli and environments have been developed (D'Zmura et al., 2000) and a $4 \mathrm{D}$ maze game is available ${ }^{2}$. As mentioned in the review of previous work, a variety of geometric approaches have been used: The projection, slicing, and rotation methods. Some studies provided additional cues to enhance $4 \mathrm{D}$ representations. New technologies, such as virtual and augmented reality, can create complex representations of $4 \mathrm{D}$, however, the broader question is rather theoretical: Human retina has a $2 \mathrm{D}$ structure onto which the environment needs to be projected. In the case of a 3D environment, the optics of the eye and the physical properties of our $3 \mathrm{D}$ environment dictate the use of projective geometry to describe how the distal stimulus is transformed into the corresponding proximal stimulus. How can a $4 \mathrm{D}$ world be projected onto $2 \mathrm{D}$ proximal stimulus? First, even though we cannot physically build 4D objects, we have mathematical languages to describe and reason about arbitrary spatial dimensions. In fact, some of the early evidence about the possibility of $4 \mathrm{D}$ representations came from some mathematicians' phenomenal reports of $4 \mathrm{D}$ perceptions during their studies of 4D spaces (Davis et al., 1995). But unlike 3D to $2 \mathrm{D}$ projections, where physical properties of the environment and the physiology of the eyes determine a specific type of projection, there is a priori no such constraint for $4 \mathrm{D}$. There are infinitely many ways of projecting a higher dimension to a lower one, and

${ }^{2}$ http://www.urticator.net/maze/ without any constraints, which one is most appropriate, remains an open question.

\section{OUTSTANDING PROBLEMS: PRACTICE OR NO PRACTICE, FEEDBACK OR NO FEEDBACK}

Perceptual learning is a complex process that depends on multiple factors such as motivation, attention, feedback, and amount of practice. Given the drastic changes it implies, learning $4 \mathrm{D}$ representations is likely to necessitate time for adaptation. However, how much time this adaptation will take is not known. Providing feedback can help accelerate this process. However, a major issue with feedback is that subjects can, consciously or unconsciously, discover low-level cues that can lead to successful responses without necessarily building $4 \mathrm{D}$ representations. This observation points to another fundamental problem, viz., how to eliminate stimulus artifacts that correlate positively with the task.

\section{OUTSTANDING PROBLEMS: ELIMINATION OF ARTIFACTS}

A major problem in studying 4D is to determine whether subjects' behavioral success can be based on cues (as mentioned above, some studies used additional cues such as color or principal vanishing points) and factors that are not inherently 4D. For example, Aflalo and Graziano (2008) used two theoretical models of path integration that were designed to solve the puzzle by using only $3 \mathrm{D}$ spatial reasoning and looked for better performance than these models to infer $4 \mathrm{D}$ capabilities. However, Wang (2014a) noted that path integration can be solved algebraically without building 4D representations. Frank (2014) and Wang (2014b) argued whether hypervolume estimation requires 4D representations. Selection of experimental variables and the design of the experiments require a careful analysis of all artifacts and alternative strategies without $4 \mathrm{D}$ representations in order to conclude that indeed humans can build 4D representations.

\section{OUTSTANDING PROBLEMS: INTER-SUBJECT VARIABILITY}

Another issue that needs further investigation is inter-subject variability, as exemplified by the discussion of Miwa et al.'s (2018) results above. It is conceivable that, because of factors such as attention and motivation, some subjects may take longer or may need different training strategies to build $4 \mathrm{D}$ representations. Given that even simple visual illusions are not universally experienced (Phillips, 2019), there is also the possibility that only a subset of humans can achieve this competence. Subject populations in these studies were relatively small and composed mainly of college students. It may be also possible that humans lose their ability of building highdimensional spaces early in childhood as observed in various "critical periods" in development. Hence, there is a need to 
further analyze inter-subject variability in order to be able to generalize the results.

\section{WHAT CAN MACHINE LEARNING TELL US ABOUT 4D REPRESENTATIONS?}

It has been shown that 3-layer neural networks are "universal approximators," in that they can learn arbitrary input-output mappings with arbitrary precision (Funahashi, 1989; Hornik et al., 1989, 1990). However, the success of these networks in solving practical problems has been severely constrained in the past by limited training data sets and limited computational power to carry out the training. Recently "deep neural networks" have shown success beyond the performance levels obtained by traditional computer vision and pattern recognition algorithms. In addition to rich data sets and enhanced computing power, this success can be traced to the fact that several layers of these networks include a priori computations (e.g., convolutions) that are aimed to capture some of the desired output properties, such as position invariance. By the "no-free lunch" (Wolpert, 1996) and "bias-variance" (Geman et al., 1992) theorems, it is clear that introducing such a priori biases to networks can limit their domain of universality while enhancing their performance in applications in which the bias matches a desired output property, e.g., position invariance. Hence one way to study how $4 \mathrm{D}$ representations can be built is through training a variety of neural network architectures, each including different a priori structures that reflect a priori biases, to find networks that can and those that cannot learn $4 \mathrm{D}$ representations.

\section{NEUROIMAGING TECHNIQUES PERTINENT TO THE PRESENCE OR ABSENCE OF SOLID 4D PERCEPTS}

\section{Decoding 4D Percepts From fMRI Signals}

As noted above, improvements in task performance in a $4 \mathrm{D}$ environment alone may not be sufficient to conclude that humans can build 4D representations due to potential artifact by factors that are not inherently $4 \mathrm{D}$. This problem persists when one is to test $4 \mathrm{D}$ representations in the brain by using neuroimaging techniques such as functional magnetic resonance imaging (fMRI). Studies for 3D perception have used electrophysiological techniques (Ohzawa et al., 1990; DeAngelis et al., 1991; Tsao et al., 2003) and fMRI (Tsao et al.,

\section{REFERENCES}

Aflalo, T. N., and Graziano, M. S. A. (2008). Four dimensional spatial reasoning in humans. J. Exp. Psychol. Hum. Percept. Perform. 34, 1066-1077. doi: 10.1037/ 0096-1523.34.5.1066

Ambinder, M. S., Wang, R. F., Crowell, J. A., and Francis, G. K. (2009). Human four-dimensional spatial intuition in virtual reality. Psychon. Bull. Rev. 16, 818-823. doi: 10.3758/PBR.16.5.818
2003; Preston et al., 2008; Orban, 2011) to identify brain areas that represent $3 \mathrm{D}$ information. These studies reported that $3 \mathrm{D}$ information can be extracted from activations in multiple visual areas along with the hierarchy of the visual system. In particular, it has been shown that a particular visual area such as $\mathrm{V} 3 \mathrm{~B} / \mathrm{KO}$ is involved in integration of disparity and motion cues to depth (Ban et al., 2012). More recently, the entorhinal cortex has been shown to be involved in representation of 3D space (Kim and Maguire, 2019). Based on these findings in studies for 3D perception, it can be hypothesized that visual areas and the entorhinal cortex come to be involved in representation and integration of $4 \mathrm{D}$ information once human subjects acquire 4D percept. More concretely, if activations in these areas accurately predict subjects' perceptual report and/or performance in $4 \mathrm{D}$ visual tasks, it will strongly support that subjects actually perceive an object in $4 \mathrm{D}$ space.

\section{CONCLUSION}

Perceiving higher spatial dimensions beyond 3D has been a longstanding quest for a broad range of researchers including but not limited to philosophers, mathematicians, psychologists, neuroscientists, developers of virtual reality, and engineers. A success in this endeavor will literally create a new dimension for the human mind. It may be possible to reach not only 4 but even higher dimensions. If such an ability exists, its training and testing pose formidable challenges. In this article, we reviewed uncertainties pertaining to whether observers truly learn and represent a 4D scene or rather rely on indirect lower dimensional cues to perform a task which is within a $4 \mathrm{D}$ visual space. This high-risk high-return topic has the potential to completely transform our understanding of brain representations and their plasticity.

\section{AUTHOR CONTRIBUTIONS}

$\mathrm{HO}$ and AY drafted the work with indeed more contribution of $\mathrm{HO}$. AY selected and organized the figure. KS provided the draft of the imaging part.

\section{FUNDING}

KS was partially supported by JSPS KAKENHI grant number 19H01041.

Ban, H., Preston, T. J., Meeson, A., and Welchman, A. E. (2012). The integration of motion and disparity cues to depth in dorsal visual cortex. Nat. Neurosci. 15, 636-643. doi: 10.1038/nn.3046

Chrastil, E. R., and Warren, W. H. (2014). From cognitive maps to cognitive graphs. PLoS One 9:e112544. doi: 10.1371/journal.pone.0112544

Davis, P. J., Hersh, R., Marchisotto, R. A., Davis, P. J., and Hersh, R. (eds) (1995). "Four dimensional intuition," in The Mathematical Experience, (Boston: Houghton Mifflin Co). 
DeAngelis, G. C., Ohzawa, I., and Freeman, R. D. (1991). Depth is encoded in the visual cortex by a specialized receptive field structure. Nature 352, 156-159. doi: $10.1038 / 352156 \mathrm{a} 0$

Dobbins, A. C., Jeo, R. M., Fiser, J., and Allman, J. M. (1998). Distance modulation of neural activity in the visual cortex. Science 281, 552-555. doi: 10.1126/ science.281.5376.552

D'Zmura, M., Colantoni, P., and Seyranian, G. (2000). Virtual environments with four or more spatial dimensions. Presence: Teleoperators and Virtual Environments 9, 616-631. doi: 10.1162/105474600300040411

Frank, A. U. (2014). Four-dimensional representation in human cognition and difficulties with demonstrations: a commentary on wang. Spat. Cogn. Comput. 14, 114-120. doi: 10.1080/13875868.2014.885526

Funahashi, K. (1989). On the approximate realization of continuous-mappings by neural networks. Neural Netw. 2, 183-192. doi: 10.1016/0893-6080(89)90003-8

Geman, S., Bienenstock, E., and Doursat, R. (1992). Neural networks and the bias/variance dilemma. Neural Comput. 4, 1-58. doi: 10.1109/72.883447

Grieves, R. M., and Jeffery, K. J. (2017). The representation of Space in the Brain. Behav. Process. 135, 113-131.

Gross, C. G., and Graziano, M. S. A. (1995). Multiple Representations of Space in the Brain. Neuroscientist 1, 43-50. doi: 10.1177/107385849500100107

Harris, C. S. (1965). Perceptual adaptation to inverted, reversed, and displaced vision. Psychol. Rev. 72, 419-444. doi: 10.1037/h0022616

Hornik, K., Stinchcombe, M., and White, H. (1989). Multilayer feedforward networks are universal approximators. Neural Netw. 2, 359-366. doi: 10.1016/ 0893-6080(89)90020-8

Hornik, K., Stinchcombe, M., and White, H. (1990). Universal approximation of an unknown mapping and its derivatives using multilayer feedforward networks. Neural Netw. 3, 551-560. doi: 10.1016/0893-6080(90)90005-6

Huggett, N. (ed.) (1999). Space from Zeno to Einstein: Classic Readings with a Contemporary Commentary. Cambridge: MIT Press.

Kim, M., and Maguire, E. A. (2019). Can we study 3D grid codes non-invasively in the human brain? Methodological considerations and fMRI findings. NeuroImage 186, 667-678. doi: 10.1016/j.neuroimage.2018.11.041

Kohler, I. (1962). Experiments with goggles. Scientific Am. 206, 62-73.

Linden, D. E., Kallenbach, U., Heinecke, A., Singer, W., and Goebel, R. (1999). The myth of upright vision. A psychophysical and functional imaging study of adaptation to inverting spectacles. Perception 28, 469-481. doi: 10.1068/ p2820

Miwa, T., Sakai, Y., and Hashimoto, S. (2018). Learning 4-D spatial representations through perceptual experience with hypercubes. IEEE Trans. Cogn. Dev. Syst. 10, 250-266. doi: 10.1109/tcds.2017.2710420

Ohzawa, I., DeAngelis, G. C., and Freeman, R. D. (1990). Stereoscopic depth discrimination in the visual cortex: neurons ideally suited as disparity detectors. Science 249, 1037-1041. doi: 10.1126/science.2396096
Olson, C. R. (2003). Brain Representation of Object-Centered Space in Monkeys and Humans. Annu. Rev. Neurosci. 26, 331-354.

Orban, G. A. (2011). The extraction of 3D shape in the visual system of human and nonhuman primates. Annu. Rev. Neurosci. 34, 361-388. doi: 10.1146/annurevneuro-061010-113819

Phillips, W. L. (2019). "Cross-Cultural Differences in Visual Perception of Color, Illusions, Depth, and Pictures," in Cross-Cultural Psychology: Contemporary Themes and Perspectives, 2nd Edn, ed. K. D. Keith, (Hoboken: John Wiley).

Piaget, J. (1936). La Naissance De Lintelligence Chez L'enfant, 7th Edn. Paris: Delachaux et Niestlé.

Piaget, J. (1950). La Construction Du Réel Chez L'enfant, 6th Edn. Paris: Delachaux et Niestlé.

Preston, T. J., Li, S., Kourtzi, Z., and Welchman, A. E. (2008). Multivoxel pattern selectivity for perceptually relevant binocular disparities in the human brain. J. Neurosci. 28, 11315-11327. doi: 10.1523/JNEUROSCI.2728-08.2008

Qian, J., and Yazdanbakhsh, A. (2015). A Neural Model of Distance-Dependent Percept of Object Size Constancy. PloS one 10:e0129377. doi: 10.1371/journal. pone. 0129377

Spelke, E. S., Dehaene, S., and Brannon, E. (eds) (2011). "Natural number and natural geometry," in Space, Time and Number in the Brain, (Oxford: Oxford University Press), 287-317. doi: 10.1016/b978-0-12-385948-8.00018-9

Stratton, G. M. (1897). Vision without inversion of the retinal image. Psych. Rev. 4, 341-360. doi: 10.1037/h0075482

Tsao, D. Y., Vanduffel, W., Sasaki, Y., Fize, D., Knutsen, T. A., Mandeville, J. B., et al. (2003). Stereopsis activates V3A and caudal intraparietal areas in macaques and humans. Neuron 39, 555-568. doi: 10.1016/s0896-6273(03)00459-8

Wang, R. F. (2014a). Human four-dimensional spatial judgments of hyper-volume. Spat. Cogn. Comput. 14, 91-113. doi: 10.1080/13875868.2013.870569

Wang, R. F. (2014b). Stronger evidence for four-dimensional spatial representations: a response to Frank. Spat. Cogn. Comput. 14, 121-123. doi: 10.1080/13875868.2014.885979

Wolpert, D. H. (1996). The lack of A priori distinctions between learning algorithms. Neural Comput. 8, 1341-1390. doi: 10.1162/neco.1996.8.7.1341

Conflict of Interest: The authors declare that the research was conducted in the absence of any commercial or financial relationships that could be construed as a potential conflict of interest.

Copyright (c) 2020 Ogmen, Shibata and Yazdanbakhsh. This is an open-access article distributed under the terms of the Creative Commons Attribution License (CC BY). The use, distribution or reproduction in other forums is permitted, provided the original author(s) and the copyright owner(s) are credited and that the original publication in this journal is cited, in accordance with accepted academic practice. No use, distribution or reproduction is permitted which does not comply with these terms. 